variable with possible covariates in multiple regression modeling.

Results The prevalence of moderate to severe depression in the PT population was $41.2 \%$. Multiple regression to predict PHQ-9 diagnosed depression based on TFI total score, clinical variables, and demographics showed a significant odds ratio for TFI (OR: 1.084, CI: 1.065-1.096, p<0.001). The increase of sub-scores for quality of life interference and emotionality showed significant increases in odds for depression (OR: 1.031, CI: 1.016-1.046, p<0.001; OR: 1.042, CI: 1.027$1.058, \mathrm{p}<0.001)$. Unemployed vs. employed and laterality were also associated with an increased odds of depression, and age with decreased odds while controlling for tinnitus score.

Moderate to severe anxiety was present in $43.1 \%$. Multiple regression analysis showed TFI was associated with increased of odds for anxiety (OR: 1.056, CI: 1.043-1.070, p<0.001). TFI sub-scores for emotionality (OR: 1.043, $\mathrm{p}<0.001$ ), quality of life (OR: 1.019, $\mathrm{p}<0.005)$, and cognitive interferences (OR: $0.983, p=0.015$ ) were found to be significantly associated with anxiety scores.

Conclusion The estimated prevalence of moderate to severe depression and anxiety in the US PT population is $41.2 \%$ and $43.1 \%$, respectively, which is higher than a recent systematic review of depression in tinnitus suggesting a depression prevalence of 33\%. TFI score was significantly associated with both depression and anxiety. Similarly, more granular sub-scores of the TFI elucidate how PT affects the psychological health of these patients.

Disclosures E. Smith: None. M. Amans: 1; C; This work was funded by an NIH grant. 2; C; Consultant for Covidien and Stryker. K. Meisel: None. D. McCoy: None.

\section{E-089 EARLY TRANSCRIPTIONAL REGULATION OF TENASCIN-C ATTENUATES POST-STROKE BRAIN DAMAGE}

${ }^{1} \mathrm{~K}$ Johnson*, ${ }^{2} \mathrm{~B}$ Chelluboina. ${ }^{1}$ Neurosurgery, University of Illinois, Peoria, IL; ${ }^{2}$, Neurosurgery, University of Wisconsin School of Medicine, Madison, WI

10.1136/neurintsurg-2019-SNIS. 164

Background Stroke induced alterations in the Extracellular matrix play a vital role in mediating acute pathogenesis as well as in post-stroke recovery. Tenascin-C (TNC), an adhesion-modulating extracellular matrix glycoprotein, its upregulation in adults is linked to vascular diseases. Recently, an elevated blood level of TNC in large artery atherosclerotic stroke patients and its association with post-stroke inflammation is reported. Based on the literature and our recent research investigation, we hypothesize that specific suppression of TNC immediately after ischemic stroke by using a non-viral shRNA attenuated the post-stroke acute brain damage.

Methods In order to attain our objectives, we utilized young adult male Sprague-Dawley rats. Selected animal groups were subjected to two-hour middle cerebral artery occlusion followed by three-day reperfusion with or without TNCshRNA treatment immediately after reperfusion. We performed various techniques including TTC, Realtime-PCR, and immunoblot and immunofluorescence analysis in order to investigate the effect of our treatments and the molecular mechanisms.
Results Our results clearly demonstrated that TNC is persistently upregulated after ischemic stroke. Specific regulation of TNC immediately after ischemia inhibited the TNC levels and significantly reduced the extent of brain damage. TNC inhibition after stroke modulated the expression of excitatory amino acid transporters and tight junction proteins. Further, Early inhibition of TNC after stroke regulated the expression of key inflammatory mediators such as Toll-Like Receptor-4 and Tumor Necrosis Factor- $\alpha$ and Matrix metalloproteinase-9.

Conclusions Based on our results we conclude that TNC mediated several acute ischemic stroke pathogenic mechanisms. Transcriptional inactivation of TNC regulated the post-stroke alterations in excitatory amino acid transporters, tight junction proteins, and inflammatory mediators and thus attenuated the post-stroke brain damage.

Disclosures K. Johnson: None. B. Chelluboina: None.

\section{E-090 GEOGRAPHIC SERVICE DELIVERY FOR ENDOVASCULAR CLOT RETRIEVAL: USING DISCRETE EVENT SIMULATION TO OPTIMISE RESOURCES}

${ }^{1} \mathrm{Y}$ Ren ${ }^{*},{ }^{2} \mathrm{M}$ Phan, ${ }^{2} \mathrm{~J}$ Maingard, ${ }^{2} \mathrm{C}$ Seah, ${ }^{2} \mathrm{~J} \mathrm{Wu},{ }^{3} \mathrm{P}$ Luong, ${ }^{2} \mathrm{D}$ Shell, ${ }^{4} \mathrm{M}$ Burney, ${ }^{2} \mathrm{~K}$ Zhou, ${ }^{1} \mathrm{~A}$ lamanna, ${ }^{5} \mathrm{H}$ Kok, ${ }^{2} \mathrm{R}$ Chandra, ${ }^{6} \mathrm{~A}$ Jhamb, ${ }^{1} \mathrm{~V}$ Thijs, ${ }^{1} \mathrm{D}$ Brooks, ${ }^{1} \mathrm{H}$ Asadi. ${ }^{1}$ A ustin health, Melbourne, AUSTRALIA; ${ }^{2}$ Monash health, Melbourne, Australia; ${ }^{3}$ RMIT, Melbourne, Australia; ${ }^{4}$ Deloitte Digital, Sydney, Australia; ${ }^{5}$ Northern Health, Melbourne, Australia; ${ }^{6}$ St Vincent's Hospital, Melbourne, Australia

\subsection{6/neurintsurg-2019-SNIS. 165}

Background Endovascular thrombectomy is the standard of care for acute ischaemic stroke caused by large vessel occlusion. Reducing stroke symptom onset to reperfusion time is associated with improved functional outcomes. In this study we aimed to develop a computational model to predict and identify time-related outcomes of community stroke calls within an area based on variable parameters.

Method A model to simulate and predict EVT service delivery at capable hospital within a geographic area, city or state was designed using SimPy, a Python based discrete event simulation framework. Geolocation data defined by the user as well as that used by the model is sourced using the Google Maps Application Programming Interface (API) and GeoPy. Variables are customized by the user based on their local environment to provide more acute prediction.

Result This algorithm can predict the delay between the time that emergency services are notified of a potential stroke and cerebral reperfusion using EVT at capable hospital. Factors including area size and population, number of EVT capable hospitals, number of available angiography machines, availability of ambulances and time of patient can be adjusted to observe the effect of modifying each parameter input.

Conclusion This model, made available as an open source web-based application, provides a mean of predicting resource utilisation and wait times for stroke service delivery planning. By modifying parameter inputs, the delivery and coordination of a stroke service within an area, city or state may be optimised and aid the development of new EVT service protocols. Disclosures Y. Ren: None. M. Phan: None. J. Maingard: None. C. Seah: None. J. Wu: None. P. Luong: None. D. Shell: None. M. Burney: None. K. Zhou: None. A. lamanna: None. H. Kok: None. R. Chandra: None. A. Jhamb: None. V. Thijs: None. D. Brooks: None. H. Asadi: None. 